\title{
Semilinear evolution equations with distributed measures
}

\author{
Adrian Petruşel $^{1 *}$ and Bianca Satco ${ }^{2}$
}

\author{
"Correspondence: \\ petrusel@math.ubbcluj.ro \\ ${ }^{1}$ Faculty of Mathematics and \\ Computer Science, Babeş-Bolyai \\ University, Cluj-Napoca, Romania \\ Full list of author information is \\ available at the end of the article
}

\begin{abstract}
The aim of the paper is to provide, by an approach based on the Mönch fixed point theorem, existence results for the semilinear evolution problem with distributed measures

$$
\left\{\begin{array}{l}
d x=(-A x+f(t, x)) d t+d g, \quad t \in[0,1] \\
x(0)=x_{0}
\end{array}\right.
$$

where $-A$ is the infinitesimal generator of a (uniformly or strongly) continuous semigroup $\{T(t), t \geq 0\}$ of bounded linear operators, $f$ is not necessarily continuous and $g:[0,1] \rightarrow X$ is a regulated function.

Working with Kurzweil-Stieltjes integrals and using a measure of non-compactness allows us to relax the assumptions on the semigroup, on $f$ and $g$ comparing to some already known results.
\end{abstract}

MSC: $37 \mathrm{~L} 05 ; 47 \mathrm{D06} ; 47 \mathrm{~J} 35 ;$ 58D25; 26A42; 47H10

Keywords: semilinear evolution problem with distributed measure; Kurzweil-Stieltjes integral; uniformly continuous semigroup; strongly continuous semigroup; mild solution; regulated function; Hausdorff measure of non-compactness; Mönch fixed point theorem; fixed point

\section{Springer}

\section{Introduction}

The aim of the paper is to provide existence results for the semilinear evolution problem with distributed measures (1), where $-A$ is the infinitesimal generator of a continuous semigroup $\{T(t), t \geq 0\}$ of bounded linear operators, $f:[0,1] \times X \rightarrow X$ and $g:[0,1] \rightarrow X$.

This problem was firstly considered in [1] for a continuous function $f$ and a bounded variation function $g,\{T(t), t \geq 0\}$ being a compact $C_{0}$ semigroup of contractions. The importance of allowing the occurrence of the very general term $d g$ on the right-hand side of the equation was clearly exposed and exemplified in [1]; the equation models situations that arise, e.g., in optimal control problems with state constraints.

We are now interested in discussing the matter of existence of mild solutions (defined in a similar way to the classical case) for the above problem under less restrictive assumptions: a regulated function $g$ (not necessarily of bounded variation) and a possibly discontinuous function $f$.

(c) 2015 Petruşel and Satco. This article is distributed under the terms of the Creative Commons Attribution 4.0 International License (http://creativecommons.org/licenses/by/4.0/), which permits unrestricted use, distribution, and reproduction in any medium, provided you give appropriate credit to the original author(s) and the source, provide a link to the Creative Commons license, and indicate if changes were made. 
At the first step, $\{T(t), t \geq 0\}$ is a uniformly continuous semigroup of bounded linear operators. The proof is based on a fixed point argument, via the Mönch fixed point theorem, using the Hausdorff measure of non-compactness. We would like to mention that our approach uses the Kurzweil-Stieltjes integral in a Banach space and this allows us to relax the assumptions on $f$ and $g$ comparing to the already known results (such as Theorem 8.1 in [1]).

An illustrating example (Example 25), of a parabolic problem with dynamic boundary conditions showing the applicability of the main result, is also described.

Finally, we discuss the more general case when $-A$ is the infinitesimal generator of a strongly continuous semigroup $\{T(t), t \geq 0\}$ of bounded linear operators. In this setting, the problem becomes complicated due to the possibility for the Stieltjes integral to be not well defined in the Banach space. Working with Kurzweil-type integration theory we are able to prove that if the semigroup has bounded $\mathcal{B}$-variation and the Banach space is reflexive, then an existence theory can be developed by the same method.

Our results extend and generalize some other recent theorems in the literature as well, see $[2,3]$ or $[4-6]$ (for the linear case).

\section{Kurzweil integration in Banach spaces}

Let $[a, b]$ be an interval of the real line equipped with the usual topology and the Lebesgue measure $d t$. Throughout this paper $X$ is a Banach space with norm $\|\cdot\|$.

Let us now introduce the definition of Kurzweil integral in Banach spaces, which is one of the possible extensions of the notion of Henstock-Kurzweil integral for real-valued functions (the reader is referred to $[7,8]$ or [9]).

A partition of $[a, b]$ is a finite collection of pairs $\left\{\left(\left[t_{i-1}, t_{i}\right], c_{i}\right), i=1, \ldots, n\right\}$, where $\left[t_{i-1}, t_{i}\right]$ are non-overlapping subintervals of $[a, b], c_{i} \in\left[t_{i-1}, t_{i}\right], i=1, \ldots, n$, and $\bigcup_{i=1}^{n}\left[t_{i-1}, t_{i}\right]=[a, b]$. A gauge $\delta$ on $[a, b]$ is a positive function on $[a, b]$. For a given gauge $\delta$, we say that a partition is $\delta$-fine if $\left[t_{i-1}, t_{i}\right] \subset\left(c_{i}-\delta\left(c_{i}\right), c_{i}+\delta\left(c_{i}\right)\right)$ for any $i \in\{1, \ldots, n\}$.

Definition 1 ([10]) A function $f:[a, b] \rightarrow X$ is said to be Kurzweil-integrable on $[a, b]$ if there exists a vector $(\mathrm{K}) \int_{a}^{b} f(s) d s \in X$ such that, for every $\varepsilon>0$, there is a gauge $\delta_{\varepsilon}$ on $[a, b]$ satisfying

$$
\left\|\sum_{i=1}^{n} f\left(c_{i}\right)\left(t_{i}-t_{i-1}\right)-(\mathrm{K}) \int_{a}^{b} f(s) d s\right\|<\varepsilon
$$

for every $\delta_{\varepsilon}$-fine partition $\left\{\left(\left[t_{i-1}, t_{i}\right], c_{i}\right), i=1, \ldots, n\right\}$ of $[a, b]$.

If $f$ is Kurzweil-integrable, then it has the same feature on any sub-interval of $[a, b]$. The function $t \mapsto(\mathrm{K}) \int_{a}^{t} f(s) d s$ is called the Kurzweil-primitive of $f$ on $[a, b]$.

Remark 2 The Kurzweil primitive is continuous (see [10]). The connection between this integral, other gauge integrals (Henstock-Lebesgue and Henstock-Kurzweil-Pettis integrals) and the classical ones (namely, Bochner and Pettis integrals) can be found in [11,12] or [13].

Endowed with the Alexiewicz-norm

$$
\|f\|_{A}=\sup _{t \in[a, b]}\left\|(\mathrm{K}) \int_{a}^{t} f(s) d s\right\|,
$$


the space $\mathcal{K}([a, b], X)$ of Kurzweil-integrable functions on $[a, b]$ is non-complete (for the particular real-valued case, see [14]).

\section{Kurzweil integration for bounded linear operators}

Let $L(X)$ be the space of bounded linear operators mapping $X$ in $X$ which is a Banach space with respect to the operator norm $\|\cdot\|_{o p}$ defined by

$$
\|A\|_{o p}=\sup _{\|x\| \leq 1}\|A x\| .
$$

The continuity in the operator norm topology of a semigroup $\{T(t), t \geq 0\}$ of bounded linear operators is called uniform continuity in [15]. Also, if for every $x \in X$ the function $t \mapsto T(t) x$ is continuous, then the semigroup is called strongly continuous (or $C_{0}$ semigroup) (see [15], p.4) or $\mathcal{B}$-continuous (by the terminology in [16] and the references therein).

Let us recall the following concepts.

\section{Definition 3}

(i) ([17] or [18]) A function $g:[a, b] \rightarrow X$ is regulated if it has at most discontinuities of the first kind on $[a, b]$, i.e., for every $t \in[a, b)$ there exists $g(t+) \in X$ such that $\lim _{s \rightarrow t, s>t}\|g(s)-g(t+)\|=0$ and for every $t \in(a, b]$ there exists $g(t-) \in X$ such that $\lim _{s \rightarrow t, s<t}\|g(s)-g(t-)\|=0$.

(ii) $([19,20])$ Likewise, an operator-valued function $T:[a, b] \rightarrow L(X)$ is regulated if for every $t \in[a, b)$ there exists $T(t+) \in L(X)$ and for every $t \in(a, b]$ there exists $T(t-) \in L(X)$ in the operator norm topology;

(iii) An operator-valued function $T:[a, b] \rightarrow L(X)$ is said to be $\mathcal{B}$-regulated ([16, 21, 22]) if $t \in[a, b] \mapsto T(t) x$ is regulated for each $x \in X$ with $\|x\| \leq 1$.

By $G([a, b], X)$ we denote the space of regulated $X$-valued functions endowed with its natural (Banach space) norm $\|f\|_{C}=\sup _{t \in[a, b]}\|f(t)\|$.

One of the main tools in our work is the following concept.

Definition 4 ([17]) A set $\mathcal{A} \subset G([a, b], X)$ is said to be equi-regulated if for every $\varepsilon>0$ and every $t_{0} \in[a, b]$ there exists $\delta>0$ such that:

(i) for any $t_{0}-\delta<t^{\prime}<t_{0}$ : $\left\|x\left(t^{\prime}\right)-x\left(t_{0}-\right)\right\|<\varepsilon$;

(ii) for any $t_{0}<t^{\prime \prime}<t_{0}+\delta$ : $\left\|x\left(t^{\prime \prime}\right)-x\left(t_{0}+\right)\right\|<\varepsilon$ for all $x \in \mathcal{A}$.

A useful version of Ascoli's theorem for regulated functions was proved in [18] (see also [17] in finite dimensional setting).

Lemma 5 Let $\mathcal{A} \subset G([a, b], X)$ be equi-regulated and, for every $t \in[a, b], \mathcal{A}(t)=\{x(t)$, $x \in \mathcal{A}\}$ be relatively compact. Then $\mathcal{A}$ is relatively compact in $G([a, b], X)$.

\section{Definition 6}

(i) A function $g:[a, b] \rightarrow X$ is of bounded variation if its total variation on $[a, b]$ is 
finite, i.e.,

$$
V_{a}^{b}(g)=\sup \left\{\sum_{i=1}^{n}\left\|g\left(t_{i}\right)-g\left(t_{i-1}\right)\right\|\right\}<\infty,
$$

where the supremum is taken over all finite partitions of the interval $[a, b]$.

(ii) Likewise, an operator-valued function $T:[a, b] \rightarrow L(X)$ is said to be of bounded variation if its total variation

$$
V_{a}^{b}(T)=\sup \left\{\sum_{i=1}^{n}\left\|T\left(t_{i}\right)-T\left(t_{i-1}\right)\right\|_{o p}\right\}
$$

is finite, where the supremum has the same meaning as above.

(iii) An operator-valued function $T:[a, b] \rightarrow L(X)$ is of $\mathcal{B}$-bounded variation (see [19]) or of bounded semi-variation (as in [6]) if

$$
(\mathcal{B}) V_{a}^{b}(T)=\sup \sup _{\left\|x_{i}\right\| \leq 1}\left\{\left\|\sum_{i=1}^{n}\left(T\left(t_{i}\right)-T\left(t_{i-1}\right)\right) x_{i}\right\|\right\}<\infty,
$$

where the supremum is taken over all finite partitions of the interval $[a, b]$.

It is well known that any bounded variation vector-valued or operator-valued function is regulated. As for the corresponding $\mathcal{B}$-notions of bounded variation and regularity, notice that if $X$ is a uniformly convex space, then all operator-valued functions of $\mathcal{B}$-bounded variation are $\mathcal{B}$-regulated (see Theorem 1 in [21]). In fact, in [23] (Theorem 5) even more was proved: that every function $T:[a, b] \rightarrow L(X)$ of $\mathcal{B}$-bounded variation is $\mathcal{B}$-regulated if and only if $X$ does not contain any copy of $c_{0}$.

Remark 7 In $[16,19]$ it is shown that for operator-valued functions the regularity (resp. bounded variation property) is stronger than the $\mathcal{B}$-regularity (resp. than $\mathcal{B}$-bounded variation property) and that they respectively coincide if $X$ is finite dimensional.

It is not difficult to check ([4] or [16]) that $(\mathcal{B}) V_{a}^{b}(\cdot)$ defines a seminorm on the linear space (with usual operations) of operator-valued functions of $\mathcal{B}$-bounded variation on $[a, b]$ and that $\|A\|_{S V}=\|A(a)\|_{o p}+(\mathcal{B}) V_{a}^{b}(A)$ is a norm on this space (that becomes, in this way, a Banach space).

Similarly, $\|A\|_{B V}^{o p}=\|A(a)\|_{o p}+V_{a}^{b}(A)$ is a (Banach space) norm on the space of operatorvalued functions of bounded variation.

The Kurzweil-Stieltjes integral for operator-valued functions is defined as follows.

Definition 8 ([5,19]) Let $T:[a, b] \rightarrow L(X)$ and $h:[a, b] \rightarrow X$.

(i) We say that $h$ is Kurzweil-Stieltjes integrable with respect to $T$ if there exists an element $\int_{a}^{b} d[T(t)] h(t) \in X$ such that for every $\varepsilon>0$ there exists a gauge $\delta_{\varepsilon}$ satisfying

$$
\left\|\sum_{i=1}^{n}\left[T\left(t_{i}\right)-T\left(t_{i-1}\right)\right] h\left(c_{i}\right)-\int_{a}^{b} d[T(t)] h(t)\right\| \leq \varepsilon
$$

for every $\delta_{\varepsilon}$-fine partition $\left\{\left(\left[t_{i-1}, t_{i}\right], c_{i}\right), i=\overline{1, n}\right\}$ of $[a, b]$; 
(ii) The operator-valued function $T$ is said to be Kurzweil-Stieltjes integrable with respect to $h$ if there exists an element $\int_{a}^{b} T(t) d h(t) \in X$ such that for every $\varepsilon>0$ there exists a gauge $\delta_{\varepsilon}$ satisfying

$$
\left\|\sum_{i=1}^{n} T\left(c_{i}\right)\left(h\left(t_{i}\right)-h\left(t_{i-1}\right)\right)-\int_{a}^{b} T(t) d h(t)\right\| \leq \varepsilon
$$

for every $\delta_{\varepsilon}$-fine partition of $[a, b]$.

If the gauge $\delta_{\varepsilon}$ in the preceding Definition 8(i) can be chosen as a positive constant, then the function $h$ is called Riemann-Stieltjes integrable with respect to $T$ (and likewise for Definition 8(ii)). Theorem 2.1 in [1] contains a particular case of Proposition 2.1 in [5]: if $T$ is uniformly continuous and $h$ is of bounded variation, then the integral $\int_{a}^{b} T(t) d h(t)$ exists as Riemann-Stieltjes integral.

Remark 9 As it can be seen in [19], Proposition 15, if $T$ is $\mathcal{B}$-regulated and of $\mathcal{B}$-bounded variation and $h:[a, b] \rightarrow X$ is regulated, the Kurzweil-Stieltjes integral $\int_{a}^{b} d[T(t)] h(t) \in X$ exists. In particular, this happens when $T$ has bounded variation.

Also, Proposition 2.1 in [5] states that if $T$ is regulated and $h$ has bounded variation, then the integral $\int_{a}^{b} T(t) d h(t)$ is well defined. The same is available if $T$ has $\mathcal{B}$-bounded variation and $h$ is regulated (Theorem 3.3 in [24]).

Remark 25 in [19] asserts that if $T$ is $(\mathcal{B})$-regulated, then the KS-primitive $\int_{a}^{\cdot} d[T(t)] h(t)$ is also regulated (and similar for $\int_{a}^{b} T(t) d h(t)$ if $h$ is regulated).

We shall need the following evaluation formulas.

\section{Proposition 10}

(i) (Proposition 10 in [19]) If the function $h:[a, b] \rightarrow X$ is Kurzweil-Stieltjes integrable with respect to the operator-valued function $T:[a, b] \rightarrow L(X)$ of $\mathcal{B}$-bounded variation, then

$$
\left\|\int_{a}^{b} d[T(t)] h(t)\right\| \leq(\mathcal{B}) V_{a}^{b}(T) \cdot\|h\|_{C}
$$

(ii) (Theorem 5.1 in [16]) If $T:[a, b] \rightarrow L(X)$ has $\mathcal{B}$-bounded variation and $h$ is regulated, then

$$
\left\|\int_{a}^{b} T(t) d h(t)\right\| \leq\left(\|T(a)\|+\|T(b)\|+(\mathcal{B}) V_{a}^{b}(T)\right) \cdot\|h\|_{C} .
$$

We now present an integration by parts theorem that comes from some related results given in [6] and [25] (it can also be proved by combining Theorem 1.15 in [26] and Corollary 3.6 in [24]).

Theorem 11 Let $T:[a, b] \rightarrow L(X)$ and $g:[a, b] \rightarrow X$ satisfy the following assumptions:

(i) $T$ is of $\mathcal{B}$-bounded variation;

(ii) $g$ is Kurzweil-integrable. 
Then Tg is also Kurzweil-integrable and

$$
(\mathrm{K}) \int_{a}^{b} T(s) g(s) d s=T(b)(\mathrm{K}) \int_{a}^{b} g(s) d s-\int_{a}^{b} d[T(t)]\left((\mathrm{K}) \int_{a}^{t} g(s) d s\right) .
$$

Proof Notice first that the primitive in Kurzweil sense $[a, b] \ni t \mapsto(\mathrm{K}) \int_{a}^{t} g(s) d s \in X$ is continuous (see [10]).

Theorem 2.3 in [6] asserts (for the specific bilinear triple $(L(X), X, X)$ ) that $T$ is RiemannStieltjes integrable with respect to (K) $\int_{a}^{t} g(s) d s$, (K) $\int_{a}^{t} g(s) d s$ is Riemann-Stieltjes integrable with respect to $T$ and that

$$
\begin{aligned}
& \int_{a}^{b} T(s) d\left((\mathrm{~K}) \int_{a}^{t} g(s) d s\right) \\
& \quad=T(b)(\mathrm{K}) \int_{a}^{b} g(s) d s-T(a)(\mathrm{K}) \int_{a}^{a} g(s) d s-\int_{a}^{b} d[T(t)]\left((\mathrm{K}) \int_{a}^{t} g(s) d s\right) .
\end{aligned}
$$

But Corollary 11 in [25] yields that the Kurzweil-Stieltjes integrability of $T$ with respect to $(\mathrm{K}) \int_{a}^{t} g(s) d s$ implies the Kurzweil integrability of $T g$ (for the map $t \in[a, b] \mapsto T g(t)=$ $T(t) g(t) \in X$ we can consider an integration in the sense described for vector functions in the previous section) and the equality

$$
\int_{a}^{b} T(s) d\left((\mathrm{~K}) \int_{a}^{t} g(s) d s\right)=(\mathrm{K}) \int_{a}^{b} T(s) g(s) d s
$$

and thus the result is proved.

Lemma 12 If $\{T(t), t \geq 0\}$ is a uniformly continuous semigroup, then $T$ is of bounded variation on any compact interval and for any $t^{\prime} \leq t^{\prime \prime}$,

$$
V_{0}^{t^{\prime}}\left(T\left(t^{\prime}-\cdot\right)-T\left(t^{\prime \prime}-\cdot\right)\right) \leq\left\|T\left(t^{\prime \prime}-t^{\prime}\right)-I\right\|_{o p} \cdot V_{0}^{t^{\prime}}\left(T\left(t^{\prime}-\cdot\right)\right)
$$

Proof Let $A$ be the infinitesimal generator of the semigroup $T(t)$ (that is a bounded linear operator, see [15]). Note that $\|T(t)\|_{o p} \leq e^{t\|A\|_{o p}}$ for all $t$. Then, for all partitions of a compact interval $[a, b]$,

$$
\begin{aligned}
\sum_{i=1}^{n}\left\|T\left(t_{i}\right)-T\left(t_{i-1}\right)\right\|_{o p} & =\sum_{i=1}^{n}\left\|T\left(t_{i-1}\right) T\left(t_{i}-t_{i-1}\right)-T\left(t_{i-1}\right)\right\|_{o p} \\
& \leq e^{b\|A\|_{o p}} \sum_{i=1}^{n}\left\|T\left(t_{i}-t_{i-1}\right)-I\right\|_{o p}
\end{aligned}
$$

and from here, since $T(t)=e^{t A}$, it follows, by an estimation of the power series, that

$$
\begin{aligned}
\sum_{i=1}^{n}\left\|T\left(t_{i}\right)-T\left(t_{i-1}\right)\right\|_{o p} & \leq e^{b\|A\|_{o p}} \sum_{i=1}^{n}\left(t_{i}-t_{i-1}\right)\|A\|_{o p} e^{\left(t_{i}-t_{i-1}\right)\|A\|_{o p}} \\
& \leq e^{b\|A\|_{o p}}\|A\|_{o p} e^{(b-a)\|A\|_{o p}}(b-a)
\end{aligned}
$$

and the bounded variation property is proved. 
Concerning the second assertion, let $t^{\prime} \leq t^{\prime \prime}$. For any partition $t_{1}<\cdots<t_{n}$ of $\left[0, t^{\prime}\right]$, we have

$$
\begin{aligned}
& \sum_{i=1}^{n}\left\|\left(T\left(t^{\prime}-t_{i}\right)-T\left(t^{\prime \prime}-t_{i}\right)\right)-\left(T\left(t^{\prime}-t_{i-1}\right)-T\left(t^{\prime \prime}-t_{i-1}\right)\right)\right\|_{o p} \\
& \quad=\sum_{i=1}^{n}\left\|\left(T\left(t^{\prime}-t_{i}\right)-T\left(t^{\prime}-t_{i-1}\right)\right)-\left(T\left(t^{\prime \prime}-t_{i}\right)-T\left(t^{\prime \prime}-t_{i-1}\right)\right)\right\|_{o p} \\
& \quad=\sum_{i=1}^{n}\left\|\left(T\left(t^{\prime}-t_{i}\right)-T\left(t^{\prime}-t_{i-1}\right)\right)-T\left(t^{\prime \prime}-t^{\prime}\right)\left(T\left(t^{\prime}-t_{i}\right)-T\left(t^{\prime}-t_{i-1}\right)\right)\right\|_{o p} \\
& \quad \leq \sum_{i=1}^{n}\left\|T\left(t^{\prime \prime}-t^{\prime}\right)-I\right\|_{o p}\left\|T\left(t^{\prime}-t_{i}\right)-T\left(t^{\prime}-t_{i-1}\right)\right\|_{o p} \\
& \quad \leq\left\|T\left(t^{\prime \prime}-t^{\prime}\right)-I\right\|_{o p} \cdot V_{0}^{t^{\prime}}\left(T\left(t^{\prime}-\cdot\right)\right),
\end{aligned}
$$

and so

$$
V_{0}^{t^{\prime}}\left(T\left(t^{\prime}-\cdot\right)-T\left(t^{\prime \prime}-\cdot\right)\right) \leq\left\|T\left(t^{\prime \prime}-t^{\prime}\right)-I\right\|_{o p} \cdot V_{0}^{t^{\prime}}\left(T\left(t^{\prime}-\cdot\right)\right) .
$$

Lemma 13 If $\{T(t), t \geq 0\}$ is a uniformly continuous semigroup and $h:[a, b] \rightarrow X$ is regulated, then $\int_{a}^{\cdot} T(\cdot-s) d h(s)$ is regulated.

Proof Applying the integration by parts Corollary 3.6 in [24], we get

$$
\int_{a}^{t} T(t-s) d h(s)+\int_{a}^{t} d[T(t-s)] h(s)=h(t)-T(t-a) h(a),
$$

and so it is sufficient to prove that $\int_{a}^{\cdot} d[T(\cdot-s)] h(s)$ is regulated. To this purpose, fix $t \in$ $[a, b)$ and let $t^{\prime}>t$ tend to $t$ (the case $t^{\prime}<t$ can be treated in the same way). Then, by Proposition 10(i),

$$
\begin{aligned}
& \left\|\int_{a}^{t^{\prime}} d\left[T\left(t^{\prime}-s\right)\right] h(s)-\int_{a}^{t} d[T(t-s)] h(s)\right\| \\
& \quad \leq\left\|\int_{a}^{t} d\left[T\left(t^{\prime}-s\right)-T(t-s)\right] h(s)\right\|+\left\|\int_{t}^{t^{\prime}} d\left[T\left(t^{\prime}-s\right)\right] h(s)\right\| \\
& \quad \leq V_{a}^{t}\left(T\left(t^{\prime}-\cdot\right)-T(t-\cdot)\right) \cdot\|h\|_{C}+V_{t}^{t^{\prime}}\left(T\left(t^{\prime}-\cdot\right)\right) \cdot\|h\|_{C} .
\end{aligned}
$$

Using Lemma 12 one gets

$$
\begin{aligned}
& \left\|\int_{a}^{t^{\prime}} d\left[T\left(t^{\prime}-s\right)\right] h(s)-\int_{a}^{t} d[T(t-s)] h(s)\right\| \\
& \quad \leq\left\|T\left(t^{\prime}-t\right)-I\right\|_{o p} \cdot V_{0}^{t}(T(t-\cdot)) \cdot\|h\|_{C}+V_{t}^{t^{\prime}}\left(T\left(t^{\prime}-\cdot\right)\right) \cdot\|h\|_{C} \\
& \quad \leq\left\|T\left(t^{\prime}-t\right)-I\right\|_{o p} \cdot V_{0}^{b}(T) \cdot\|h\|_{C}+V_{t}^{t^{\prime}}\left(T\left(t^{\prime}-\cdot\right)\right) \cdot\|h\|_{C} .
\end{aligned}
$$


As in the first part of the proof of Lemma 12, it can be shown that $V_{t}^{t^{\prime}}\left(T\left(t^{\prime}-\cdot\right)\right) \leq$ $e^{b\|A\|_{o p}}\|A\|_{o p} e^{\left(t^{\prime}-t\right)\|A\|_{o p}}\left(t^{\prime}-t\right)$, and so $V_{t}^{t^{\prime}}\left(T\left(t^{\prime}-\cdot\right)\right) \rightarrow 0$ when $t^{\prime} \rightarrow t$, and so the regularity property is immediate.

We end this section with a mean value result that comes in an obvious manner (taking into account that the integral is a limit of integral sums).

Lemma 14 Let $T:[a, b] \rightarrow L(X)$ and $g:[a, b] \rightarrow X$ be such that Tg is Kurzweil-integrable. Then, for each $t \in[a, b]$,

(K) $\int_{a}^{t} T(s) g(s) d s \in(t-a) \overline{\operatorname{conv}}(T([a, t]) g([a, t]))$.

\section{Solutions for semilinear evolution equations with distributed measures using Kurzweil-Stieltjes integration}

Our goal is to obtain an existence result for the semilinear evolution problem with distributed measures

$$
\left\{\begin{array}{l}
d x=(-A x+f(t, x)) d t+d g, \quad t \in[0,1] \\
x(0)=x_{0}
\end{array}\right.
$$

where $-A$ is the infinitesimal generator of a uniformly continuous semigroup $\{T(t), t \geq 0\}$ of bounded linear operators, $g:[0,1] \rightarrow X$ is a regulated function, and $x_{0} \in X$.

We begin by clarifying the concept of solution that we are searching for. Notice that, in the sequel, the integrals are taken in Kurzweil sense.

Definition 15 A function $x:[0,1] \rightarrow X$ is called a $\mathcal{L}^{\infty}$-solution on $[0, \alpha]$ of this problem if it is a regulated solution of the integral equation

$$
x(t)=T(t) x_{0}+(\mathrm{K}) \int_{0}^{t} T(t-s) f(s, x(s)) d s+(\mathrm{K}) \int_{0}^{t} T(t-s) d g(s), \quad t \in[0, \alpha] .
$$

Remark that the functions on the right-hand side of the equality in this definition are regulated as a consequence of Corollary 18 and Lemma 13.

Remark 16 When the above integrals exist in Riemann, respectively Riemann-Stieltjes sense (e.g., when $f$ is continuous and $g$ has bounded variation), we get the notion of solution used in [1] (p.3200).

If, moreover, $d g=G d t$ is defined by a density $G \in L^{1}([0,1], X)$ (e.g., when $X$ has the Radon-Nikodym property and $g$ is absolutely continuous), the previous definition concerns classical mild solutions.

In order to simplify the proof of the main theorem, we first present some auxiliary results.

Lemma 17 Let $\mathcal{G}$ be a family of $X$-valued Kurzweil-integrable functions on $[a, b]$ such that the set of their primitives is equi-continuous, and let $\{T(t), t \geq 0\}$ be a uniformly continuous semigroup. Then the family $\left\{(\mathrm{K}) \int_{a}^{\cdot} T(\cdot-s) g(s) d s, g \in \mathcal{G}\right\}$ is equi-continuous on $[a, b]$. 
Proof By hypothesis, the collection of primitives of functions in $\mathcal{G}$ is $\|\cdot\|_{C}$-bounded (by $M$ ).

Fix now $t_{2} \in[a, b]$ and let $t_{1} \in\left[a, t_{2}\right]$. Applying Theorem 11 we obtain

$$
\begin{aligned}
& \left\|(\mathrm{K}) \int_{a}^{t_{1}} T\left(t_{1}-s\right) g(s) d s-(\mathrm{K}) \int_{a}^{t_{2}} T\left(t_{2}-s\right) g(s) d s\right\| \\
& \leq\left\|(\mathrm{K}) \int_{a}^{t_{1}}\left(T\left(t_{1}-s\right)-T\left(t_{2}-s\right)\right) g(s) d s\right\|+\left\|(\mathrm{K}) \int_{t_{1}}^{t_{2}} T\left(t_{2}-s\right) g(s) d s\right\| \\
& \leq\left\|\left(I-T\left(t_{2}-t_{1}\right)\right)(\mathrm{K}) \int_{a}^{t_{1}} g(s) d s\right\|+\left\|\int_{a}^{t_{1}} d\left[T\left(t_{1}-t\right)-T\left(t_{2}-t\right)\right](\mathrm{K}) \int_{a}^{t} g(s) d s\right\| \\
& \quad+\left\|(\mathrm{K}) \int_{t_{1}}^{t_{2}} g(s) d s\right\|+\left\|\int_{t_{1}}^{t_{2}} d\left[T\left(t_{2}-t\right)\right](\mathrm{K}) \int_{t_{1}}^{t} g(s) d s\right\| .
\end{aligned}
$$

From Proposition 10 it follows that

$$
\begin{aligned}
&\left\|(\mathrm{K}) \int_{a}^{t_{1}} T\left(t_{1}-s\right) g(s) d s-(\mathrm{K}) \int_{a}^{t_{2}} T\left(t_{2}-s\right) g(s) d s\right\| \\
& \leq\left\|I-T\left(t_{2}-t_{1}\right)\right\|_{o p} \cdot\left\|(\mathrm{K}) \int_{a}^{t_{1}} g(s) d s\right\|+(\mathcal{B}) V_{a}^{t_{1}}\left(T\left(t_{1}-\cdot\right)-T\left(t_{2}-\cdot\right)\right) \cdot\|g\|_{A} \\
& \quad+\left\|(\mathrm{K}) \int_{t_{1}}^{t_{2}} g(s) d s\right\|+(\mathcal{B}) V_{a}^{t_{2}}\left(T\left(t_{2}-\cdot\right)\right) \cdot \sup _{t \in\left[t_{1}, t_{2}\right]}\left\|(\mathrm{K}) \int_{t_{1}}^{t} g(s) d s\right\|
\end{aligned}
$$

and, by Lemma 12, that

$$
\begin{aligned}
\|(\mathrm{K}) & \int_{a}^{t_{1}} T\left(t_{1}-s\right) g(s) d s-(\mathrm{K}) \int_{a}^{t_{2}} T\left(t_{2}-s\right) g(s) d s \| \\
\leq & \left(\left\|I-T\left(t_{2}-t_{1}\right)\right\|_{o p}+\left\|T\left(t_{2}-t_{1}\right)-I\right\|_{o p} \cdot V_{a}^{t_{1}}\left(T\left(t_{1}-\cdot\right)\right)\right) \cdot M \\
& +\left(1+V_{a}^{t_{2}}\left(T\left(t_{2}-\cdot\right)\right)\right) \cdot \sup _{t \in\left[t_{1}, t_{2}\right]}\left\|(\mathrm{K}) \int_{t_{1}}^{t} g(s) d s\right\| \\
\quad \leq & \left\|I-T\left(t_{2}-t_{1}\right)\right\|_{o p}\left(1+V_{0}^{b-a}(T)\right) \cdot M+\left(1+V_{0}^{b-a}(T)\right) \cdot \sup _{t \in\left[t_{1}, t_{2}\right]}\left\|(\mathrm{K}) \int_{t_{1}}^{t} g(s) d s\right\|
\end{aligned}
$$

that can be made less than some positive $\varepsilon$ for $t_{1}$ close to $t_{2}$ (independently of $g \in \mathcal{G}$ ).

For a singleton $\mathcal{G}$, we get the following.

Corollary 18 If $g:[a, b] \rightarrow X$ is Kurzweil-integrable and $T:[a, b] \rightarrow L(X)$ as in the preceding lemma, then $(\mathrm{K}) \int_{a}^{\cdot} T(\cdot-s) g(s) d s$ is continuous.

Since we allow the occurrence of discontinuous functions, we shall use instead a measure of non-compactness. Recall that the Hausdorff measure of non-compactness $\beta_{H}$ is defined, for any $A \subset X$, by the infimum of all $r>0$ such that there exists a finite number of balls covering $A$, of radius smaller than $r$ (we refer the reader to [27] or [28]). 
Lemma 19 Let $\{T(t), t \geq 0\}$ be a strongly continuous semigroup of bounded linear operators. Then, for every bounded $M \subset X$ and any interval $[a, b]$,

$$
\beta_{H}(T([a, b]) M) \leq \sup _{t \in[a, b]}\|T(t)\|_{o p} \cdot \beta_{H}(M) .
$$

Proof Note first that for each $t \in[a, b], T(t)$ maps a ball $B(\tilde{x}, r) \subset X$ into the ball $B\left(T(t) \tilde{x}, r\|T(t)\|_{o p}\right)$ since

$$
\|T(t) x-T(t) \tilde{x}\| \leq\|T(t)\|_{o p} \cdot\|x-\tilde{x}\|, \quad \forall x \in B(\tilde{x}, r)
$$

Let now $r$ be a positive number such that the set $M$ can be covered by a finite number of balls of radius (smaller than) $r$, centered at $x_{1}, \ldots, x_{N} \in X$, and let $\varepsilon>0$.

Since $t \mapsto T(t) x_{i}, i=1, \ldots, N$, are, by Corollary 2.3 in [15], continuous maps (therefore uniformly continuous), there exists $\delta_{\varepsilon}$ such that $\left|t^{\prime}-t^{\prime \prime}\right|<\delta_{\varepsilon}$ implies

$$
\left\|T\left(t^{\prime}\right) x_{i}-T\left(t^{\prime \prime}\right) x_{i}\right\|<\varepsilon, \quad \forall i=1, \ldots, N
$$

We choose $t_{1}^{\varepsilon}, \ldots, t_{N_{\varepsilon}}^{\varepsilon} \in[a, b]$ with distance less than $\delta_{\varepsilon}$ between them and to points $a$ and $b$.

Taking now an arbitrary $t$, there exists $t_{j}^{\varepsilon} \in[a, b]$ such that $\left|t-t_{j}^{\varepsilon}\right|<\delta_{\varepsilon}$. As noted, $T(t) M$ can be covered by the balls of radius smaller than $r\|T(t)\|_{o p}$ centered at $T(t) x_{1}, \ldots, T(t) x_{N}$. So, for each $x \in X$, one can find $x_{i}, i \in\{1, \ldots, N\}$, such that

$$
\left\|T(t) x-T(t) x_{i}\right\| \leq r\|T(t)\|_{o p} \leq r \sup _{t \in[a, b]}\|T(t)\|_{o p} .
$$

It follows that

$$
\left\|T(t) x-T\left(t_{j}^{\varepsilon}\right) x_{i}\right\| \leq\left\|T(t) x-T(t) x_{i}\right\|+\left\|T(t) x_{i}-T\left(t_{j}^{\varepsilon}\right) x_{i}\right\|<r \sup _{t \in[a, b]}\|T(t)\|_{o p}+\varepsilon
$$

Therefore $T([a, b]) M$ is contained in the union of balls centered at the points $\left\{T\left(t_{j}^{\varepsilon}\right) x_{i}, j=\right.$ $\left.1, \ldots, N_{\varepsilon}, i=1, \ldots, N\right\}$, of radius smaller than $r \sup _{t \in[a, b]}\|T(t)\|_{o p}+\varepsilon$, which yields that

$$
\beta_{H}(T([a, b]) M) \leq \sup _{t \in[a, b]}\|T(t)\|_{o p} \cdot \beta_{H}(M) .
$$

The Mönch fixed point theorem that we recall below will be the main tool in obtaining the existence result.

Theorem 20 ([29]) Let $D$ be a closed, convex subset of a Banach space and $N: D \rightarrow D$ be continuous with further property that for some $x_{0} \in D$ one has: $C \subset D$ countable, $\bar{C}=$ $\overline{\operatorname{conv}}\left(\left\{x_{0}\right\} \cup N(C)\right) \Longrightarrow \bar{C}$ compact. Then $N$ has a fixed point.

We proceed now to presenting the main result of the paper.

Theorem 21 Let $-A$ be the infinitesimal generator of a uniformly continuous semigroup $\{T(t), t \geq 0\}, g:[0,1] \rightarrow X$ be regulated and $f:[0,1] \times X \rightarrow X$ satisfy the following conditions: 
(1) for every $x \in G([0,1], X)$, the mapping $f(\cdot, x(\cdot))$ is Kurzweil-integrable and the function

$$
x \in G([0,1], X) \mapsto f(\cdot, x(\cdot)) \in \mathcal{K}([0,1], X)
$$

is $\|\cdot\|_{A}$-continuous;

(2) the family $\left\{(\mathrm{K}) \int_{0} f(s, x(s)) d s, x \in G([0,1], X)\right\}$ is equi-continuous;

(3) there exists a constant $c>0$ such that $\beta_{H}(f([0,1] \times D)) \leq c \beta_{H}(D)$ for every bounded $D \subset X$.

Then the evolution problem (1) has at least one $\mathcal{L}^{\infty}$-solution on some non-zero length interval $[0, \alpha] \subset[0,1]$.

Proof By Lemma 17 we get the equi-continuity of the set

$$
\left\{(\mathrm{K}) \int_{0} T(\cdot-s) f(s, x(s)) d s, x \in G([0,1], X)\right\},
$$

and so, by Lemma 13, the equi-regularity of

$$
\left\{(\mathrm{K}) \int_{0}^{\cdot} T(\cdot-s) f(s, x(s)) d s+(\mathrm{K}) \int_{0}^{\cdot} T(\cdot-s) d g(s), x \in G([0,1], X)\right\} .
$$

Thus, for some fixed $M>0$, there exists a positive $\alpha$ with the property that

$$
\begin{aligned}
& \left\|(\mathrm{K}) \int_{0}^{t} T(t-s) f(s, x(s)) d s+(\mathrm{K}) \int_{0}^{t} T(t-s) d g(s)\right\| \leq M, \\
& \forall t \in[0, \alpha], \forall x \in G([0,1], X) .
\end{aligned}
$$

Besides, $A$ is a bounded linear operator and

$$
\|T(t)\|_{o p} \leq e^{t\|A\|_{o p}}, \quad \forall t \in[0,1]
$$

Obviously, we can choose $\alpha$ such that $\alpha e^{\alpha\|A\|_{o p}} c<1$.

We shall prove that the preceding evolution problem has at least one $\mathcal{L}^{\infty}$-solution on $[0, \alpha]$.

Consider the closed convex set

$$
\mathcal{K}=\left\{x \in G([0, \alpha], X),\|x(t)\| \leq M+e^{\alpha\|A\|_{o p}}\left\|x_{0}\right\|, \forall t \in[0, \alpha]\right\}
$$

By definition, $\mathcal{L}^{\infty}$-solution for our problem means solution of the integral equation

$$
x(t)=T(t) x_{0}+(\mathrm{K}) \int_{0}^{t} T(t-s) f(s, x(s)) d s+(\mathrm{K}) \int_{0}^{t} T(t-s) d g(s),
$$

and so it suffices to prove that the Nemitsky operator

$$
N: \mathcal{K} \rightarrow \mathcal{K}, \quad N x(t)=T(t) x_{0}+(\mathrm{K}) \int_{0}^{t} T(t-s) f(s, x(s)) d s+(\mathrm{K}) \int_{0}^{t} T(t-s) d g(s)
$$


possesses fixed points. Note first of all that, by Corollary 18 and Lemma 13, the values of $N$ are in $G([0, \alpha], X)$, while the choice of $\alpha$ ensures that for all $x \in \mathcal{K}$ and all $t \in[0, \alpha]$,

$$
\|(N x)(t)\| \leq M+e^{\alpha\|A\|_{o p}}\left\|x_{0}\right\|,
$$

which certifies that $N$ is well defined.

We assert that the Mönch fixed point result can be applied in this case.

Indeed, for the continuity of $N$, we use Theorem 11:

$$
\begin{aligned}
\left\|N x_{1}-N x_{2}\right\|_{C}= & \sup _{t \in[0, \alpha]}\left\|(\mathrm{K}) \int_{0}^{t} T(t-s)\left(f\left(s, x_{1}(s)\right)-f\left(s, x_{2}(s)\right)\right) d s\right\| \\
\leq & \sup _{t \in[0, \alpha]}\left\|(\mathrm{K}) \int_{0}^{t} f\left(s, x_{1}(s)\right)-f\left(s, x_{2}(s)\right) d s\right\| \\
& +\sup _{t \in[0, \alpha]}\left\|\int_{0}^{t} d[T(t-s)](\mathrm{K}) \int_{0}^{s} f\left(\tau, x_{1}(\tau)\right)-f\left(\tau, x_{2}(\tau)\right) d \tau\right\| \\
\leq & \left(1+\sup _{t \in[0, \alpha]} V_{0}^{t}(T(t-\cdot))\right)\left\|f\left(\cdot, x_{1}(\cdot)\right)-f\left(\cdot, x_{2}(\cdot)\right)\right\|_{A} \\
\leq & \left(1+V_{0}^{\alpha}(T)\right)\left\|f\left(\cdot, x_{1}(\cdot)\right)-f\left(\cdot, x_{2}(\cdot)\right)\right\|_{A}
\end{aligned}
$$

whence the continuity of $N$.

Let us now prove that for an arbitrary $\bar{x} \in \mathcal{K}$, any countable collection $C \subset \mathcal{K}$ satisfying the equality $\bar{C}=\overline{\operatorname{conv}}(\{\bar{x}\} \cup N(C))$ is relatively compact. We shall apply Lemma 5 .

Let $C$ be such a subset of $\mathcal{K}$. Then $N(C)$ is equi-regulated by Lemma 17, therefore all we have to check is that for every $t \in[0, \alpha], C(t)$ is relatively compact in $X$.

To this aim, one can see (using Lemma 14 together with the properties of the measure of non-compactness) that

$$
\begin{aligned}
\beta_{H}(C(t)) & =\beta_{H}(\bar{C}(t))=\beta_{H}(\{\bar{x}(t)\} \cup N(C)(t)) \\
& =\beta_{H}(N(C)(t))=\beta_{H}(\{(N x)(t), x \in C\}) \\
& =\beta_{H}\left(\left\{T(t) x_{0}+(\mathrm{K}) \int_{0}^{t} T(t-s) f(s, x(s)) d s+(\mathrm{K}) \int_{0}^{t} T(t-s) d g(s), x \in C\right\}\right) \\
& =\beta_{H}\left(\left\{(\mathrm{~K}) \int_{0}^{t} T(t-s) f(s, x(s)) d s, x \in C\right\}\right) \\
& \leq \beta_{H}(t \overline{\operatorname{conv}}(T([0, t]) f([0, t] \times C([0, t])))) .
\end{aligned}
$$

Remark that the hypothesis of Lemma 19 is satisfied and, applying it, we obtain that

$$
\beta_{H}(C(t)) \leq t e^{t\|A\|_{o p}} \beta_{H}(f([0, t] \times C([0, t]))),
$$

and so, by assumption (3),

$$
\beta_{H}(C(t)) \leq t e^{t\|A\|_{o p}} c \beta_{H}(C([0, t])) \leq \alpha e^{\alpha\|A\|_{o p}} c \beta_{H}(C([0, t])) .
$$

It follows that

$$
\beta_{H}(C([0, t])) \leq \alpha e^{\alpha\|A\|_{o p}} c \beta_{H}(C([0, t]))
$$


and, since $\alpha e^{\alpha\|A\|_{o p}} c<1$, it is immediate that $\beta_{H}(C([0, t]))=0$. Of course it implies that $\beta_{H}(C(t))=0$ (so $C(t)$ is relatively compact) and then the Ascoli type theorem Lemma 5 applies to yield the relative compactness of $C$.

Finally, the Mönch fixed point theorem asserts that the operator $N$ possesses fixed points, which means that our evolution problem has $\mathcal{L}^{\infty}$-solutions.

Remark 22 Theorem 2.4 in [4] presents a situation where $f$ satisfies the integrability of superpositions $f(\cdot, x(\cdot))$ in hypothesis $(1)$, namely when $f$ is a Carathéodory function perturbed by a Kurzweil-integrable one. In fact, it is not difficult to see that in this case conditions (1) and (2) are both satisfied.

In particular, we deduce Theorem 8.1 in [1] as follows.

Corollary 23 Iff is continuous and $g$ has bounded variation, then the evolution problem (1) has $\mathcal{L}^{\infty}$-solutions (involving Riemann integral) on some non-empty interval.

Remark 24 Our study applied in the particular case $d g=0$ gives a result more general than those in [2] or [3] (when the measure driving the equation is the Lebesgue measure), see also [30].

We complete this section by giving an example (borrowed from [1]) in order to illustrate the applicability of our result.

Example 25 Let $\Omega$ be a bounded domain in $\mathbb{R}^{n}$ with boundary $\Gamma$ of class $C^{\infty}$ and such that $\Omega$ is locally on one side of its boundary. Consider the parabolic problem

$$
\begin{cases}d x+\left(-\Delta x+\alpha_{1}(t, x)\right) d t=d \eta & \text { in } Q_{T}, \\ d x+\left(x_{v}+\alpha_{2}(t, x)\right) d t=d \psi & \text { on } \Sigma_{T}, \\ x(0)=x_{0}^{\Omega} & \text { in } \Omega, \\ x(0)=x_{0}^{\Gamma} & \text { in } \Gamma\end{cases}
$$

where $Q_{T}=(0, T) \times \Omega, \Sigma_{T}=(0, T) \times \Gamma, \alpha_{1}, \alpha_{2}:[0, T] \times \mathbb{R} \rightarrow \mathbb{R}, x_{0}^{\Gamma} \in L^{2}(\Gamma), x_{0}^{\Omega} \in L^{2}(\Omega), x_{v}$ is the conormal derivative of $x$ at points of $\Gamma$, while $\phi, \psi:[0, T] \rightarrow L^{2}(\Gamma)$ and $\eta:[0, T] \rightarrow$ $L^{2}(\Omega)$.

Theorem 21 allows us to get the existence of $\mathcal{L}^{\infty}$-solutions when $\alpha_{1}$ and $\alpha_{2}$ are of the form of a sum of a Carathéodory function with a Kurzweil-integrable one and $\phi, \psi, \eta$ are regulated.

This can be done following the same steps as in the proof of Theorem 9.2 in [1] (that describe the way that our problem can be rewritten as a Cauchy problem of type (1)) and applying our main result. Finally, let us remark that here the conditions are weaker than those in the existence Theorem 9.2 in [1], which assumes that $\alpha_{1}$ and $\alpha_{2}$ are continuous and $\phi, \psi, \eta$ have bounded variation.

\section{Remarks on the same problem for strongly continuous semigroup}

We are facing now the matter of existence of solutions when $-A$ is the infinitesimal generator of a strongly continuous semigroup $\{T(t), t \geq 0\}$ of bounded linear operators. 
The starting point is the discussion presented in [1], p.3184 for bounded variation function $g$ : when working with Riemann-Stieltjes integral, in order that the integral $\int_{a}^{b} T(t-$ $s) d g(s)$ be well defined in $X$, some extra assumptions must be imposed on $X$, on $g$ or on the semigroup (in general, the integral is an element of the sequential completion $X^{c}$ of $X$ in the $\sigma\left(X, X^{*}\right)$ topology). More precisely, $\int_{a}^{b} T(t-s) d g(s) \in X$ if $X$ is reflexive (Remark 2.2), or if the semigroup is uniformly continuous (Theorem 2.1).

When considering the Kurzweil-Stieltjes integral, even for regulated function $g$, this problem can be solved for non-reflexive spaces and non-uniformly continuous semigroups: as recalled in Remark 9, if the semigroup has bounded $\mathcal{B}$-variation and $g$ is regulated, then the Kurzweil-Stieltjes integral $\int_{a}^{t} T(t-s) d g(s) \in X$.

As for our method of study, it can be extended to strongly continuous semigroups if we require that the Banach space $X$ is reflexive. Indeed, a closer look reveals that only Lemma 12, Lemma 13 and Lemma 17 need to be generalized. Thus:

Lemma 26 If $\{T(t), t \geq 0\}$ is a strongly continuous semigroup with bounded $\mathcal{B}$-variation on compact intervals, then $(\mathcal{B}) V_{0}^{t^{\prime}}\left(T\left(t^{\prime \prime}-\cdot\right)-T\left(t^{\prime}-\cdot\right)\right)$ tends to 0 as $t^{\prime \prime}>t^{\prime} \rightarrow t^{\prime}$.

Proof Let $t^{\prime} \leq t^{\prime \prime}$. Then, for any partition $t_{1}<\cdots<t_{n}$ of $\left[0, t^{\prime}\right]$ and all $\left\|x_{i}\right\| \leq 1$,

$$
\begin{aligned}
& \left\|\sum_{i=1}^{n}\left(T\left(t^{\prime}-t_{i}\right)-T\left(t^{\prime \prime}-t_{i}\right)\right)-\left(T\left(t^{\prime}-t_{i-1}\right)-T\left(t^{\prime \prime}-t_{i-1}\right)\right) x_{i}\right\| \\
& \quad=\left\|\sum_{i=1}^{n}\left(T\left(t^{\prime}-t_{i}\right)-T\left(t^{\prime}-t_{i-1}\right)\right)-\left(T\left(t^{\prime \prime}-t_{i}\right)-T\left(t^{\prime \prime}-t_{i-1}\right)\right) x_{i}\right\| \\
& \quad=\left\|\left(T\left(t^{\prime \prime}-t^{\prime}\right)-I\right) \sum_{i=1}^{n}\left(T\left(t^{\prime}-t_{i}\right)-T\left(t^{\prime}-t_{i-1}\right)\right) x_{i}\right\| .
\end{aligned}
$$

Now take into account that the semigroup is also weakly continuous ([15], p.44). Since for all partitions and all $\left\|x_{i}\right\| \leq 1,\left\|\sum_{i=1}^{n}\left(T\left(t^{\prime}-t_{i}\right)-T\left(t^{\prime}-t_{i-1}\right)\right) x_{i}\right\| \leq(\mathcal{B}) V_{0}^{t^{\prime}}\left(T\left(t^{\prime}-\cdot\right)\right)$, we deduce that the set $\left\{\sum_{i=1}^{n}\left(T\left(t^{\prime}-t_{i}\right)-T\left(t^{\prime}-t_{i-1}\right)\right) x_{i}\right\}$ is contained in a ball in $X$ which is weakly compact. So, for any $\varepsilon>0$, there exists $\delta_{\varepsilon}>0$ s.t. $t^{\prime \prime}-t^{\prime}<\delta_{\varepsilon}$ implies that $\|\left(T\left(t^{\prime \prime}-\right.\right.$ $\left.\left.t^{\prime}\right)-I\right) \sum_{i=1}^{n}\left(T\left(t^{\prime}-t_{i}\right)-T\left(t^{\prime}-t_{i-1}\right)\right) x_{i} \|<\varepsilon$, and so the $(\mathcal{B}) V_{0}^{t^{\prime}}\left(T\left(t^{\prime \prime}-\cdot\right)-T\left(t^{\prime}-\cdot\right)\right)$ tends to 0 as $t^{\prime \prime} \rightarrow t^{\prime}$.

Lemma 27 If $h:[a, b] \rightarrow X$ is regulated and $\{T(t), t \geq 0\}$ is a strongly continuous semigroup with bounded $(\mathcal{B})$-variation on compacts such that $(\mathcal{B}) V_{t}^{t^{\prime}}(T) \rightarrow 0$ whenever $t^{\prime} \rightarrow t$, then $\int_{a}^{\cdot} T(\cdot-s) d h(s)$ is regulated.

Proof Let us consider $t \in[a, b)$ and let $t^{\prime}>t$ tend to $t$ (the case $t^{\prime}<t$ can be treated similarly). One can write

$$
\begin{aligned}
& \int_{a}^{t^{\prime}} T\left(t^{\prime}-s\right) d h(s)-\int_{a}^{t} T(t-s) d h(s) \\
& \quad=\int_{a}^{t} T\left(t^{\prime}-s\right)-T(t-s) d h(s)+\int_{t}^{t^{\prime}} T\left(t^{\prime}-s\right) d h(s) .
\end{aligned}
$$


Now let us see that each of these terms has limit when $t^{\prime} \rightarrow t$. Concerning the first integral, a usual calculus (by passing through integral sums, e.g., [24], p.371) gives us

$$
\begin{aligned}
& \left\|\int_{a}^{t} T\left(t^{\prime}-s\right)-T(t-s) d h(s)-\left(\left(T\left(t^{\prime}-t\right)-I\right) h(t)-\left(T\left(t^{\prime}-a\right)-T(t-a)\right) h(a)\right)\right\| \\
& \quad \leq(\mathcal{B}) V_{a}^{t}\left(T\left(t^{\prime}-\cdot\right)-T(t-\cdot)\right) \cdot\|h\|_{C}
\end{aligned}
$$

which tends to 0 (by Lemma 26) and, thanks to the strong continuity of $T$, the integral has limit.

As for the second integral, from a similar calculus it comes that

$$
\begin{aligned}
& \left\|\int_{t}^{t^{\prime}} T\left(t^{\prime}-s\right) d h(s)-\left(h\left(t^{\prime}\right)-T\left(t^{\prime}-t\right) h(t)\right)\right\| \\
& \quad \leq(\mathcal{B}) V_{t}^{t^{\prime}}\left(T\left(t^{\prime}-\cdot\right)\right)\|h\|_{C},
\end{aligned}
$$

which tends to 0 by hypothesis, therefore the second integral has limit as well since $h$ is regulated and $T$ is strongly continuous.

Lemma 28 Let $\mathcal{G}$ be a family of X-valued Kurzweil-integrable functions on $[a, b]$ such that the set of their primitives is equi-continuous. Suppose that $\{T(t), t \geq 0\}$ is a strongly continuous semigroup with bounded $\mathcal{B}$-variation on $[a, b]$. Then the family $\left\{(\mathrm{K}) \int_{a}^{\cdot} T(\cdot-\right.$ $s) g(s) d s, g \in \mathcal{G}\}$ is equi-continuous.

Proof The collection of primitives of functions in $\mathcal{G}$ is $\|\cdot\|_{C}$-bounded.

Fix now $t_{2} \in[a, b]$ and let $t_{1} \in\left[a, t_{2}\right]$. By Theorem 11 ,

$$
\begin{aligned}
\|(\mathrm{K}) & \int_{a}^{t_{1}} T\left(t_{1}-s\right) g(s) d s-(\mathrm{K}) \int_{a}^{t_{2}} T\left(t_{2}-s\right) g(s) d s \| \\
\leq & \left\|\left(I-T\left(t_{2}-t_{1}\right)\right)(\mathrm{K}) \int_{a}^{t_{1}} g(s) d s\right\|+\left\|\int_{a}^{t_{1}} d\left[T\left(t_{1}-t\right)-T\left(t_{2}-t\right)\right](\mathrm{K}) \int_{a}^{t} g(s) d s\right\| \\
& +\left\|(\mathrm{K}) \int_{t_{1}}^{t_{2}} g(s) d s\right\|+\left\|\int_{t_{1}}^{t_{2}} d\left[T\left(t_{2}-t\right)\right](\mathrm{K}) \int_{t_{1}}^{t} g(s) d s\right\| \\
\leq & \left\|\left(I-T\left(t_{2}-t_{1}\right)\right)(\mathrm{K}) \int_{a}^{t_{1}} g(s) d s\right\|+(\mathcal{B}) V_{a}^{t_{1}}\left(T\left(t_{1}-\cdot\right)-T\left(t_{2}-\cdot\right)\right) \cdot\|g\|_{A} \\
& +\left\|(\mathrm{K}) \int_{t_{1}}^{t_{2}} g(s) d s\right\|+(\mathcal{B}) V_{t_{1}}^{t_{2}}\left(T\left(t_{2}-\cdot\right)\right) \cdot \sup _{t \in\left[t_{1}, t_{2}\right]}\left\|(\mathrm{K}) \int_{t_{1}}^{t} g(s) d s\right\| \\
\leq & \left\|\left(I-T\left(t_{2}-t_{1}\right)\right)(\mathrm{K}) \int_{a}^{t_{1}} g(s) d s\right\|+(\mathcal{B}) V_{a}^{t_{1}}\left(T\left(t_{1}-\cdot\right)-T\left(t_{2}-\cdot\right)\right) \cdot\|g\|_{A} \\
& +\left\|(\mathrm{K}) \int_{t_{1}}^{t_{2}} g(s) d s\right\|+(\mathcal{B}) V_{0}^{b-a}(T) \cdot \sup _{t \in\left[t_{1}, t_{2}\right]}\left\|(\mathrm{K}) \int_{t_{1}}^{t} g(s) d s\right\| .
\end{aligned}
$$

It can be made less than some positive $\varepsilon$ for $t_{1}$ close to $t_{2}$ (independently of $g \in \mathcal{G}$ ). Indeed, only the first term of the second line from below needs a reasoning similar to that in the previous proposition: the set of all (K) $\int_{a}^{t_{1}} g(s) d s, g \in \mathcal{G}$ is contained in some ball, there- 
fore in a weakly compact set, and since the semigroup is weakly continuous, the assertion follows in an obvious way.

In the described framework (of strongly continuous semigroup of bounded linear operators on a reflexive space) the main result is given below.

Theorem 29 Let $-A$ be the infinitesimal generator of a strongly continuous semigroup $\{T(t), t \geq 0\}$ with bounded $\mathcal{B}$-variation on compact intervals such that $(\mathcal{B}) V_{t}^{t^{\prime}}(T) \rightarrow 0$ whenever $t^{\prime} \rightarrow t$.

Let $g:[0,1] \rightarrow X$ be regulated and $f:[0,1] \times X \rightarrow X$ satisfy the hypotheses (1), (2), (3) in Theorem 21. Then the evolution problem (1) has at least one $\mathcal{L}^{\infty}$-solution on some non-zero length interval $[0, \alpha] \subset[0,1]$.

Proof Applying Lemma 28 brings us to the equi-continuity of the set $\left\{(\mathrm{K}) \int_{0}^{\cdot} T(\cdot-\right.$ s) $f(s, x(s)) d s, x \in G([0,1], X)\}$ whence, by Lemma 27 , to the equi-regularity of

$$
\left\{(\mathrm{K}) \int_{0}^{\cdot} T(\cdot-s) f(s, x(s)) d s+(\mathrm{K}) \int_{0}^{\cdot} T(\cdot-s) d g(s), x \in G([0,1], X)\right\} .
$$

Thus, for some fixed $M>0$, there exists a positive $\alpha$ with the property that

$$
\begin{aligned}
& \left\|(\mathrm{K}) \int_{0}^{t} T(t-s) f(s, x(s)) d s+(\mathrm{K}) \int_{0}^{t} T(t-s) d g(s)\right\| \leq M, \\
& \forall t \in[0, \alpha], \forall x \in G([0,1], X) .
\end{aligned}
$$

As $\{T(t), t \geq 0\}$ is a strongly continuous semigroup, by Theorem 2.2 at p.4 in [15], there exist $\omega \geq 0$ and $M_{T} \geq 1$ such that

$$
\|T(t)\| \leq M_{T} e^{\omega t}, \quad \forall t \geq 0 .
$$

Obviously, we can choose $\alpha$ such that $\alpha M_{T} e^{\omega \alpha} c<1$.

We shall prove that the preceding evolution problem has at least one $\mathcal{L}^{\infty}$-solution on $[0, \alpha]$.

Consider the closed convex set

$$
\mathcal{K}=\left\{x \in G([0, \alpha], X),\|x(t)\| \leq M+M_{T} e^{\omega \alpha}\left\|x_{0}\right\|, \forall t \in[0, \alpha]\right\}
$$

It suffices to prove that the operator

$$
N: \mathcal{K} \rightarrow \mathcal{K}, \quad N x(t)=T(t) x_{0}+(\mathrm{K}) \int_{0}^{t} T(t-s) f(s, x(s)) d s+(\mathrm{K}) \int_{0}^{t} T(t-s) d g(s)
$$

possesses fixed points. Note first of all that by Corollary 18 and Remark 9, the values of $N$ are in $G([0, \alpha], X)$, while the choice of $\alpha$ ensures that for all $x \in \mathcal{K}$ and all $t \in[0, \alpha]$, $\|(N x)(t)\| \leq M+M_{T} e^{\omega \alpha}\left\|x_{0}\right\|$, which certifies that $N$ is well defined.

We assert that the Mönch fixed point result is suitable in this case. 
Indeed, the continuity of $N$ comes from Theorem 11:

$$
\begin{aligned}
\left\|N x_{1}-N x_{2}\right\|_{C} & =\sup _{t \in[0, \alpha]}\left\|(\mathrm{K}) \int_{0}^{t} T(t-s)\left(f\left(s, x_{1}(s)\right)-f\left(s, x_{2}(s)\right)\right) d s\right\| \\
& \leq\left(1+\sup _{t \in[0, \alpha]}(\mathcal{B}) V_{0}^{t}(T(t-\cdot))\right)\left\|f\left(\cdot, x_{1}(\cdot)\right)-f\left(\cdot, x_{2}(\cdot)\right)\right\|_{A} \\
& \leq\left(1+(\mathcal{B}) V_{0}^{\alpha}(T)\right)\left\|f\left(\cdot, x_{1}(\cdot)\right)-f\left(\cdot, x_{2}(\cdot)\right)\right\|_{A} .
\end{aligned}
$$

Let us show that for an arbitrary $\bar{x} \in \mathcal{K}$, any countable collection $C \subset \mathcal{K}$ satisfying the equality $\bar{C}=\overline{\operatorname{conv}}(\{\bar{x}\} \cup N(C))$ is relatively compact. As in Theorem 21 , all we have to check is that for every $t \in[0, \alpha], C(t)$ is relatively compact in $X$.

Again, one can see that

$$
\beta_{H}(C(t)) \leq \beta_{H}(t \overline{\operatorname{conv}}(T([0, t]) f([0, t] \times C([0, t]))))
$$

and remark that the hypothesis of Lemma 19 is satisfied; applying it, together with (3), we get

$$
\begin{aligned}
\beta_{H}(C(t)) & \leq t M_{T} e^{\omega t} \beta_{H}(f([0, t] \times C([0, t]))) \\
& \leq t M_{T} e^{\omega t} c \beta_{H}(C([0, t])) \leq \alpha M_{T} e^{\omega \alpha} c \beta_{H}(C([0, t])) .
\end{aligned}
$$

It follows that

$$
\beta_{H}(C([0, t])) \leq \alpha M_{T} e^{\omega \alpha} c \beta_{H}(C([0, t]))
$$

and, since $\alpha M_{T} e^{\omega \alpha} c<1$, it is immediate that $\beta_{H}(C([0, t]))=0$. Of course it implies that $\beta_{H}(C(t))=0$ (so $C(t)$ is relatively compact), and then the Mönch fixed point theorem asserts that the operator $N$ possesses fixed points, which means that our evolution problem has $\mathcal{L}^{\infty}$-solutions.

\section{Conclusions}

We obtained the existence of mild solutions (in a generalized sense) for the semilinear evolution problem with distributed measures (1) under less restrictive assumptions comparing to similar results in literature, where the involved semigroup of bounded linear operators was a compact $C_{0}$ semigroup, the function $f$ in the semilinear part was continuous, and $g$ was supposed to have bounded variation.

More precisely, in a general Banach space the existence result works for a uniformly continuous semigroup (Theorem 21), while for a reflexive Banach space the existence of solutions was proved for a $C_{0}$ semigroup with bounded $(\mathcal{B})$-variation on compact intervals (Theorem 29). In both situations, the function $f$ is allowed to be discontinuous and the function $g$ is only regulated (possibly with unbounded variation).

The authors declare that they have no competing interests. 


\section{Author details}

${ }^{1}$ Faculty of Mathematics and Computer Science, Babeş-Bolyai University, Cluj-Napoca, Romania. ${ }^{2}$ Faculty of Electrical Engineering and Computer Science, Ştefan Cel Mare University, Suceava, Romania.

\section{Acknowledgements}

The first author thanks the Visiting Professor Programming at King Saud University for funding this work. The author extends his sincere appreciation to the Deanship of Scientific Research at King Saud University for its funding this Prolific Research Group (PRG-1436-10). For the second author, this work was supported by a grant of the Romanian National Authority for Scientific Research, CNCS - UEFISCDI, project number PN-II-RU-TE-2012-3-0336.

\section{Received: 24 February 2015 Accepted: 3 August 2015 Published online: 19 August 2015}

\section{References}

1. Vrabie, l: Compactness of the solution operator for a linear evolution equation with distributed measures. Trans. Am. Math. Soc. 354(8), 3181-3205 (2002)

2. Boucherif, A, Precup, R: Semilinear evolution equations with nonlocal initial conditions. Dyn. Syst. Appl. 16, 507-516 (2007)

3. Cao, Y, Sun, J: Existence of solutions for semilinear measure driven equations. J. Math. Anal. Appl. 425(2), 621-631 (2015)

4. Schwabik, Š: Generalized Ordinary Differential Equations. World Scientific, Singapore (1992)

5. Monteiro, GA, Tvrdý, M: Generalized linear differential equations in a Banach space: continuous dependence on a parameter. Discrete Contin. Dyn. Syst. 33, 283-303 (2013)

6. Bianconi, R, Federson, M: A Fredholm-type theorem for linear integral equations of Stieltjes type. Cadernos de Matematica 10, 233-269 (2009)

7. Kurzweil, J: Generalized ordinary differential equations and continuous dependence on a parameter. Czechoslov. Math. J. 7, 618-646 (1957)

8. Gordon, RA: The Integrals of Lebesgue, Denjoy, Perron and Henstock. Grad. Stud. in Math. (1994)

9. Turdý, M: Differential and integral equations in the space of regulated functions. Habil. Thesis, Praha (2001)

10. Cao, SS: The Henstock integral for Banach valued functions. Southeast Asian Bull. Math. 16, 36-40 (1992)

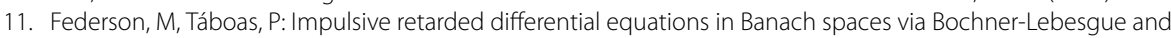
Henstock integrals. Nonlinear Anal., Theory Methods Appl. 50, 389-407 (2002)

12. Heikkilä, S, Kumpulainen, M, Seikkala, S: Convergence theorems for HL integrable vector-valued functions with applications. Nonlinear Anal., Theory Methods Appl. 70, 1939-1955 (2009)

13. Di Piazza, L, Satco, B: A new result on impulsive differential equations involving non-absolutely convergent integrals. J. Math. Anal. Appl. 352, 954-963 (2009)

14. Bongiorno, B: Relatively weakly compact sets in the Denjoy space. J. Math. Study 27, 37-43 (1994)

15. Pazy, A: Semigroups of Linear Operators and Applications to Partial Differential Equations. Applied Mathematical Sciences, vol. 44. Springer, New York (1983)

16. Monteiro, GA: On functions of bounded semivariation (21 Jan 2015). arXiv:1501.05196v1 [math.CA]

17. Fraňková, D: Regulated functions. Math. Bohem. 116(1), 20-59 (1991)

18. Mesquita, JG: Measure functional differential equations and impulsive functional dynamic equations on time scales. Ph.D. Thesis

19. Schwabik, క̌: Abstract Perron-Stieltjes integral. Math. Bohem. 121, $425-447$ (1996)

20. Schwabik, Š: A note on integration by parts for abstract Perron-Stieltjes integrals. Math. Bohem. 126(3), 613-629 (2001)

21. Barbanti, L: Simply regulated functions and semivariation in uniformly convex spaces. Real Anal. Exch. 24(1), 405-409 (1998/99)

22. Hönig, CS: Equations intégrales generalisées et applications. In: Harmonic Analysis: Study Group on Translation-Invariant Banach Spaces. Pub. Math. d'Orsay (1983)

23. Blasco, O, Gregori, P, Calabuig, JM: Finite semivariation and regulated functions by means of bilinear maps. Real Anal. Exch. 26(2), 1-6 (2000/2001)

24. Monteiro, GA, Tvrdý, M: On Kurzweil-Stieltjes integral in Banach space. Math. Bohem. 137, 365-381 (2012)

25. Federson, M: Substitution formulas for the Kurzweil and Henstock vector integrals. Math. Bohem. 127(1), 15-26 (2002)

26. Hönig, CS: There is no natural Banach-space norm on the space of Kurzweil-Henstock-Denjoy-Perron integrable functions. Seminario Brasileiro de Analise 30, 387-397 (1989)

27. Mitchell, AA, Smith, C: An existence theorem for weak solutions of differential equations in Banach spaces. In: Nonlinear Equations in Abstract Spaces (Proc. Internat. Sympos. Univ. Texas, Arlington, 1977), pp. 387-403. Academic Press, New York (1978)

28. Ambrosetti, A: Un teorema di existenza per le equazioni differenziali negli spazi di Banach. Rend. Semin. Mat. Univ. Padova 39, 349-361 (1967)

29. Mönch, H: Boundary value problems for nonlinear ordinary differential equations of second order in Banach spaces. Nonlinear Anal. 4, 985-999 (1980)

30. Dong, QX, Li, G: Measure of noncompactness and semilinear nonlocal functional differential equations in Banach spaces. Acta Math. Sin. Engl. Ser. 31(1), 140-150 (2015) 